\title{
The Effectiveness of Group Dialectical Behavior Therapy on Borderline Personality Disorder: A Systematic Review
}

\author{
Zhenyu Cai ${ }^{1, \dagger}$, Jiesen Huang ${ }^{2, \dagger}$, You $\mathrm{Wu}^{3, \dagger}$, Yinhao $\mathrm{Zhu}^{4, *}{ }^{*}$, \\ ${ }^{1}$ University of Durham,Durham, UK \\ ${ }^{2}$ Semir International School, WenZhou, Zhejiang Province, China \\ ${ }^{3}$ Guanghua Qidi International School, Shanghai Province, China \\ ${ }^{4}$ University of Washington, Seattle, USA \\ ${ }^{*}$ Corresponding author. Email: yinhaz@uw.edu \\ Those authors contributed equally.
}

\begin{abstract}
For decades, countless psychoanalysts made an effort for the exploration of this special type of psychic disorder-borderline personality disorder (BPD). More importantly, one special form of treatment, Dialectical Behavior Therapy (DBT) was found to be relatively effective in medicating this mental disease. By screening of sources retrieved from multiple databases, this review utilized and evaluated the findings of 12 studies for its clinical impact. Echoing the previous studies, DBT has shown an effectiveness in treating BPD. From mothers to female teenagers, different age groups among populations were put into considerations. Moreover, evidence is also indicating a better performance for DBT with the combination of Cognitive Therapy. Limitations include problems with sampling for each study, leading to a less generalized finding. Certain amount of individual bias could also be presented throughout this review. To conclude, BPD has shown its potential in treating BPD's symptomatology while more further research is needed in regard to limitations.
\end{abstract}

Keywords: borderline personality disorder, dialectical behaviour therapy, systematic review, group treatment

\section{INTRODUCTION}

Borderline personality disorder (BPD) is defined by the DSM-5 as "a pervasive pattern of instability of interpersonal relationships, self-image, and affects, as well as marked impulsivity, beginning in early adulthood and present in a variety of contexts [1]". Symptoms of BPD include its extreme fear of abandonment and paranoid thoughts. Some other related features include impulsive and risky behavior, fragile self-image, unstable and intense relationships, up and down moods (often as a reaction to interpersonal stress), suicidal behavior or threats of self-injury, persistent feelings of emptiness, frequent and intense displays of anger. The presence of suicide proneness, impulsivity, disturbed relationships, and affective dysregulation are all commonly seen in borderline personality disorder. It is estimated that $1.6 \%$ of the population was diagnosed with BPD [2]. In the meanwhile, it comorbids with other psychiatric disorders. About 20\% of inpatients in psychiatric clinics are diagnosed with a borderline personality disorder. Among these BPD patients, $70 \%$ of them have engaged in self-harm behaviors, and BPD patients on the average tend to attempt suicide three times than their counterparts in a lifetime [3]. This is because it shares many comorbidities with other mental illnesses or disorders, such as anxiety or particular mood disorders [4]. To conclude, borderline personality disorder is a severe psychiatric disorder as it sabotages one's mental health, physical health, as well as their normal social-functioning. Without a proper and effective treatment, patients usually would make it to the path of self-destruction. Fortunately, it has been discovered that Dialectical Behavior Therapy (DBT) is suggested to be effectively helpful in treating the symptoms of BPD [5]. 
Dialectical behaviour therapy "is a multi-module psychological intervention that was developed using some of the principles of $\mathrm{CBT}$ in combination with mindfulness-based techniques [6]. The length of the treatment is usually over a year and its mechanism is mainly using mindfulness and acceptance to regulate and control emotions and behaviours [7]. There are three main principles of DBT [8]: First as a holistic dialectical philosophy, second as an encapsulation of the opposites, and third as a continuous movement of balancing the patients and the therapists. DBT was initially an outpatient treatment, with sessions each week and skills group training. In times of emergency, individual therapists are provided, too. Treatment includes four formats: group therapy, individual psychotherapy, phone calls and consultation team meetings. Group therapy is advantageous because it allows patients to practice their problem-solving skills and social skills in a form of roleplaying within the group. These group sessions can help BPD patients to build their individual boundaries and cope with the world in a more mentalized way.

Despite the promises of group DBT in BPD patients, the effectiveness of group DBT remains unclear. Specifically, few systematic reviews or meta-analyses were found to aggregate the evidence of group DBT among BPD patients. This paper suggests that such a systematic review can help establish the empirical evidence of group DBT in BPD patients and further facilitate clinical practice.

In the current study, this paper investigates the effectiveness of DBT on BPD symptoms, especially on self-harming behaviours and negative emotions in patients with BPD, and to provide an evidence-based basis for the formulation and improvement of clinical treatment protocols.

\section{METHODOLOGY}

The methodology of this paper was mainly based on the literature review, by using google scholar as a main database of searching resources that are relevant to evaluate the effectiveness of Dialectical Behaviour Therapy (DBT) on Borderline Personality Disorder. By utilizing google scholar as a basis, more databases were used in this research. As we put search terms into these databases, they help us filter all the important information we need. For example, PsycINFO, PubMed, EBSCO were broadly used for this literature review. During this process, PsycINFO especially provided us with a convenient function in refining relative search options.

The search terms for this literature review were indicative of Borderline Personality Disorder, Dialectical Behaviour Therapy, and Group intervention. A combination of these key terms when using the "AND" option in the database setting helped us find the most appropriate and relative resource.

Also, to quantify our research resources, the Snowballing method was used in our literature review. It means that many articles were found in the reference list of one general article so that additional resources were identified. It supplemented the search results from the databases mentioned above in case relevant articles were missing.

The records retrieved from the databases were analysed based on several criteria. First, all studies need to specifically examine the effectiveness of group DBT on BPD. Second, BPD was established by a commonly used diagnostic system (e.g., DSM, ICD); Third, all references were ideally to be peer-reviewed journals and were reports of primary analysis of treatment effect.

Among the 55 collected articles from the various databases, a number of articles were screened by four of our group members independently in order to gather the most useful information and filter those which cannot be chosen as eligible evidence. After the screening, 12 studies were identified and selected features of these articles were summarized and discussed in the current study.

\section{RESULTS}

\subsection{Effects of DBT on the specific population diagnosed with $B P D$}

\subsubsection{Mother}

Group DBT has been implemented in mothers and showed an appropriate effect. A group treatment program of DBT targeted a total of 45 women, who are mothers of infants younger than three years of age and who were clinically determined to meet full or partial diagnostic criteria for borderline personality disorder (BPD). Results of this innovative adaptation of motherinfant dialectical behavior therapy (MIDBT) revealed a significant improvement in the maternal BPD symptoms and caregiver-infant connections. Especially, significant improvements in mood, anxiety levels, borderline symptoms, and reflective functioning suggest that mental health and parental metallization were improving continually [9].

A group training program conducted by Renneberg and Rosenbach (2016) also showed that DBT is helpful in the treatment of mothers with BPD. The program is based on cognitive-behavioral principles and skills derived from Dialectical Behavior Therapy. A total of 15 mothers with BPD who had young children (aged 0-6 years) participated in a 12-week training program. Results show that DBT was also created for mothers with BPD to assist them to improve their parenting abilities and nurturing their children. DBT is effective, with 
respondents reporting visible changes in their behavior and attitudes toward parenting [10].

\subsubsection{Female juvenile offenders.}

Group DBT intervention has been shown to be useful in treating female juvenile offenders with emotional problems. A collaborative project examined the effectiveness of a DBT intervention on two units of a state juvenile rehabilitation facility for female juvenile offenders. Pre-post intervention records were compared for female offenders from mental health and a general population unit where DBT was implemented. Before and after the intervention period, staff punitive responses to youth behaviour problems were compared. Female offenders had a significant decrease in serious behavioural issues during the 10-month study period, according to the findings [11].

\subsection{Effectiveness of DBT on emotional regulation and non-suicidal injury}

DBT training method has been found useful in improving emotional regulation. A current study targeted at 136 participants diagnosed with heterogeneous mental health conditions (BPD included) were enrolled in the group on a rolling basis. Their emotion regulations were assessed at the beginning and end of every six- to the eight-week module. DBT skills-training module refers to mindfulness, distress tolerance, interpersonal effectiveness, and emotion regulation, all of which have been linked to improved emotion regulation. Besides, results showed significant improvements in psychological distress, depression, borderline symptomatology, and functional impairment. There were reduced numbers of Mental Health presentations and admissions between six months pre- and post-group [12].

A randomized, controlled pilot study has supported that group DBT skills help reduce negative emotional symptoms as well. In this experiment, a total of 26 adults with bipolar I or II illness were randomized to either intervention or control groups and filled out the Beck depression inventory, mindfulness-based self-efficacy scale, and affective control scale at baseline after 12 weeks. In a series of 12 weekly 90-min sessions, participants learned DBT skills, mindfulness techniques, and general BPD psychoeducation. Results show that group DBT helps reduce depressive symptoms, improve affective control and mindfulness self-efficacy [13].

Group DBT also has been shown to be useful in reducing non-suicidal self-injury (NSSI). A total of 84 participants with BPD who self-harm were randomized either to DBT skills training or to a waitlist control group to examine the effect of DBT on NSSI during 20-weeks of Dialectical Behaviour Therapy (DBT) skills training. Results show that training in DBT skills reduced the frequency of NSSI. There was a significant effect of training in DBT skills on the incidence of NSSI [12].

\subsection{Effectiveness of combined treatment of DBT and other interventions}

First, the combination of group DBT and cognitive therapy group (CTG) was suggested to be more effective in treating patients with borderline personality disorder. An overall sample of 82 college students with BPD who attempted to suicide within the previous six months were randomly assigned to DBT or CTG. As a result of the intervention and 6-month follow-ups, both groups saw a reductions in suicide reattempts and depression. Meanwhile, cognitive bias showed improvement in the CTG, while acceptance scores in DBT and suppression scores dropped in the DBT [14].

In comparison to DBT alone, combination of MBT with DBT may improve specific aspects of skills related to social cognition and attachment stability. In a pilot study, the effectiveness of MBT given alongside DBT was evaluated on an inpatient sample with BPD, compared to DBT alone. Results showed that in comparison to DBT alone, MBT in addition to DBT improved more on social cognition and attachment stability. Self-harm was also reduced more when DBT and MBT are used together than when DBT is used alone. DBT combined with MBT may result in a decrease in frightened attachment and an increase in emotional mentalizing [15].

Group DBT has been proved to be more effective than individual DBT in patients with BPD. A preliminary study which examined the efficacy of individual DBT in 37 BPD patients, compared with Group DBT in 14 BPD patients, includes 12 months of treatment and an 18month follow-up period. Results showed that Group Dialectical behaviour therapy has proven to be an effective treatment in borderline personality disorder. However, the effectiveness in BPD of DBT skills training (DBT-ST) alone is not known [16].

\section{DISCUSSION}

The current review systematically examined the available evidence of the effectiveness of group DBT on borderline symptomatology. Overall, aggregated from various research designs and samples, group DBT was found in 12 studies effective. Multiple groups of population were identified covering mothers of young children, juvenile offenders and multiple outcomes including emotion regulation and self-harm which are core to the BPD patients.

From the current review, it is found that group DBT can be implemented in a quite wide range of the population. First, group DBT is effective in the treatment of mothers with BPD. The innovative adaptation of 
mother-infant DBT demonstrated considerable enhancement in maternal BPD symptoms and caregiverinfant connections [9]. A group training program DBT associated with cognitive-behavioural principles has helped mothers improve their childcare abilities [10]. Second, group DBT intervention is also effective on female juvenile offenders who have psychological issues. There was a continuous reduction of youth behaviour problems and staff punishments over a 10-month intervention [11]. Third, the incidence of non-suicidal self-injury (NSSI) that happened on self-harm individuals has been reduced [12].

Our results corroborated previous studies on BPD. Our results indicated that DBT may be applied to those individuals with subthreshold BPD symptoms and DBT can alleviate trans diagnostic emotional problems, for example, the difficulty of emotion regulation. Our results found that DBT not only could improve emotion status but also reduce the risk of being self-harmed. This effect could help patients in a significant way by coping with the impulse of hurting themselves and leaving more room to have a chance to change.

Our results also showed that in combination with other interventions, the treatment effect of DBT was enlarged and would be more beneficial. DBT worked effectively in minimizing depression or recurrent suicide attempts for BPD patients as well as Cognitive Therapy Group (CTG). Moreover, patients performed better in cognition error scales in the CTG group and reported lower scores in suppression scores and a rise in acceptance in the DBT group. The result suggested that the combination of DBT and CTG intervention can be successfully conducted on BDP patients [14]. The combined group of DBT and MBT decreased the severity of symptoms, like self-harm, compared to DBT alone. And short-term of this combined treatment towards amelioration of fearful attachment and mentalization [15]. Our results collectively suggested that group DBT performs not inferior to the individual DBT, more over it could have potential to perform better than the individual one due to its provision of the mimic social context that can facilitate patients to build their own personality boundaries. Despite that, the effectiveness of DBT skills training (DBT-ST) alone in $\mathrm{BPD}$ is inconclusive and requires more evidence in the future research [16].

Although group DBT has been proved to be effective in treating patients with BPD, there are still some aspects of concern. At a college counselling service, a pilot study has been conducted on 17 college students with BPD using short-term DBT, revealing a reduction in symptoms of depression and BPD symptoms and an increase in adaptive coping skills such as problemsolving and constructive self-talk, but no reduction in anxiety [17]. This peculiar result suggests that DBT may work better on some certain outcomes rather than others.
Nevertheless, considering the limited number of participants in this study, more research is needed in the future to confirm these findings.

Our current review identified that in some institutions, treating borderline personality disorder (BPD) patients with normal DBT can be problematic due to legislative or financial limitations [18]. Therefore, more research is still required to inform the clinical practice that in what situation DBT can be used independently on the treatment of BPD patients.

Our review has some limitations. First, the number of patients included in this review is limited and may introduce a certain amount of bias to the experimental results. Different research designs other than RCT with small sample size could inflate the treatment effectiveness. In addition, the patients included in the current review mostly were from western countries which could limit the generalization of the findings from our review.

\section{CONCLUSION}

There are some future directions on the investigation of the DBT that it deems as potential. The developments of mother-infant Dialectical Behavior Therapy (MIDBT) can be considered to include other systematic motherinfant therapy groups in the future, for example, systematic dyadic work or consistent referral. The adaptations of other MIDBT are used to ascertain that the mother's behavioural patterns and comprehension of her infant's requirements are sufficient to alter her infant's attachment status [9]. The effectiveness of the DBT training program ought to be analyzed. A large, randomized trial with strict controls should be conducted to evaluate the training's effects, including many factors (children's development and psychopathology, psychological distress, maternal parenting skills and attitudes, mother-child interaction patterns). Furthermore, systematic observation and analysis about mother-children interaction should be taken into consideration [10]. Future research can be about the maximum efficacy of combination intervention and the additive efficacy of a supplemented program on depression, suicide attempts, and other consequences in BPD [14].

In conclusion, group dialectical behavioral therapy exhibits full potential to treat borderline personality disorders in various populations. More research of good quality and enough power are still needed to confirm the evidence and provide more insights into this field.

\section{REFERENCES}

[1] Association, A. P. (2013). Diagnostic and statistical manual of mental disorders (DSM-5®). American Psychiatric Pub. 
[2] Torgersen $\mathrm{S}$ : The nature (and nurture) of personality disorders. Scand J Psychol 50(6):624$632,2009$.

[3] Soloff, P. H., Lis, J. A., Kelly, T., Cornelius, J., \& Ulrich, R. (1994). Risk factors for suicidal behavior in borderline personality disorder. American Journal of Psychiatry, 151(9), 1316-1323.

[4] Mehlum, L. (2009). Clinical challenges in the assessment and management of suicidal behaviour in patients with borderline personality disorder. Epidemiologia e Psichiatria Sociale, 18(3), 184 190.

[5] O'Connell, B., \& Dowling, M. (2014). Dialectical behaviour therapy (DBT) in the treatment of borderline personality disorder. Journal of Psychiatric and Mental Health Nursing, 21(6), 518525.

[6] Stoffers-Winterling, J. M., Voellm, B. A., Rücker, G., Timmer, A., Huband, N., \&

Lieb, K. (2012). Psychological therapies for people with borderline personality disorder. Cochrane Database of Systematic Reviews, (8), CD005652.

[7] Lynch T.R., Chapman A.L., Rosenthal M.Z., et al. (2006) Mechanisms of change in dialectical behavior therapy: theoretical and empirical observations. Journal of Clinical Psychology 62, 459-480.

[8] Linehan M.M. (1993a) Cognitive Behavioural Treatment for Borderline Personality Disorder. Guilford Press, New York.

[9] Williams, Anne E. Sved, et al. "A new therapeutic group to help women with borderline personality disorder and their infants." Journal of Psychiatric Practice ${ }^{\circledR} 24.5$ (2018): 331-340.

[10] Renneberg, Babette, and Charlotte Rosenbach. "“There is not much help for mothers like me": Parenting Skills for Mothers with Borderline Personality Disorder-a newly developed group training program." Borderline personality disorder and emotion dysregulation 3.1 (2016): 1-7.

[11] Trupin, Eric W., et al. "Effectiveness of a dialectical behaviour therapy program for incarcerated female juvenile offenders." Child and Adolescent Mental Health 7.3 (2002): 121-127.

[12] Heath, Nicole, et al. "Group-based DBT skills training modules are linked to independent and additive improvements in emotion regulation in a heterogeneous outpatient sample." Psychotherapy Research (2021): 1-11.
[13] Van Dijk, Sheri, Janet Jeffrey, and Mark R. Katz. "A randomized, controlled, pilot study of dialectical behavior therapy skills in a psychoeducational group for individuals with bipolar disorder." Journal of affective disorders 145.3 (2013): 386-393.

[14] Lin, Tsung-Jen, et al. "The effectiveness of dialectical behavior therapy skills training group vs. cognitive therapy group on reducing depression and suicide attempts for borderline personality disorder in Taiwan." Archives of Suicide Research23.1 (2019): 82-99.

[15] Edel, Marc-Andreas, et al. "Exploring the effectiveness of combined mentalization-based group therapy and dialectical behaviour therapy for inpatients with borderline personality disorder-A pilot study." British Journal of Clinical Psychology56.1 (2017): 1-15.

[16] Andión, Óscar, et al. "Effectiveness of combined individual and group dialectical behavior therapy compared to only individual dialectical behavior therapy: A preliminary study." Psychotherapy 49.2 (2012): 241.

[17] Stratton, Natalie, et al. "Predictors of dropout from a 20-week dialectical behavior therapy skills group for suicidal behaviors and borderline personality disorder." Journal of personality disorders 34.2 (2020): 216-230. 